DOI: 10.1002/adma.((please add manuscript number))

\title{
Ultra-broadband polarisers based on metastable free-standing aligned carbon nanotube membranes
}

By Matthew T. Cole*, Matthew Doherty, Richard Parmee, Paul Dawson, \& William I. Milne

Dr. M. T. Cole, Mr. R. Parmee, \& Prof. W. I. Milne

Department of Engineering, Electrical Engineering Division,

University of Cambridge, 9 JJ Thomson Avenue, CB3 OFA, Cambridge (UK)

E-mail: mtc35@cam.ac.uk

Dr. M. Doherty, \& Prof. P. Dawson

Centre for Nanostructured Media,

Queen's University Belfast, BT7 1NN, Belfast (UK)

E-mail: p.dawson@qub.ac.uk

Prof. W. I. Milne

Department of Information Display,

Kyung Hee University, 130-701, Seoul (Republic of Korea)

E-mail: wim1@cam.ac.uk

* Corresponding author: mtc35@cam.ac.uk

Keywords: multi-walled carbon nanotubes; chemical vapour deposition; membrane; broadband optical polarisor; horizontal alignment

Exploiting the anisotropic properties of aligned one-dimensional carbon nano-allotropes has proven useful in myriad applications, including: cold cathode field emission ${ }^{[1,}{ }^{2]}$, nanostructured ropes ${ }^{[3,}{ }^{4]}$, nanoelectromechanical switched capacitive memory ${ }^{[5]}$, $\operatorname{composites}^{[6-8]}$ and birefringent media ${ }^{[9,10]}$. For many applications, it is important, however; that the native opto-electronic properties of the aligned nanostructured media remain unmodified by the device fabrication processes. By investigating the chemical vapour deposition conditions of multi-walled carbon nanotubes we have developed a route to produce highly aligned free-standing carbon nanotube films by engineering a metastable, membrane-like morphology. Using 
an augmented Leonard-Jones formalism we elucidate the conditions required for membrane formation which we show depends critically on the nanotube length and packing density, dictated by the detailed growth conditions, in addition to entropic contributions associated with the detailed membrane morphology. We demonstrate an ultra-broadband polarising behaviour of our binder- and substrate-less membranes, investigated in the wavelength range from $400 \mathrm{~nm}$ to $2.5 \mathrm{~mm}$. Extinction ratios of up to $6.4 \mathrm{~dB}$ are measured, consistent with effective medium and full numerical simulations using index and absorption constants extracted from the Drude-Lorentz model. The free-standing metastable membranes represent a new paradigm in the design and fabrication of optical components in emerging supercontinuum light sources based on sub-wavelength periodic nanomaterials.

\section{ToC Figure}
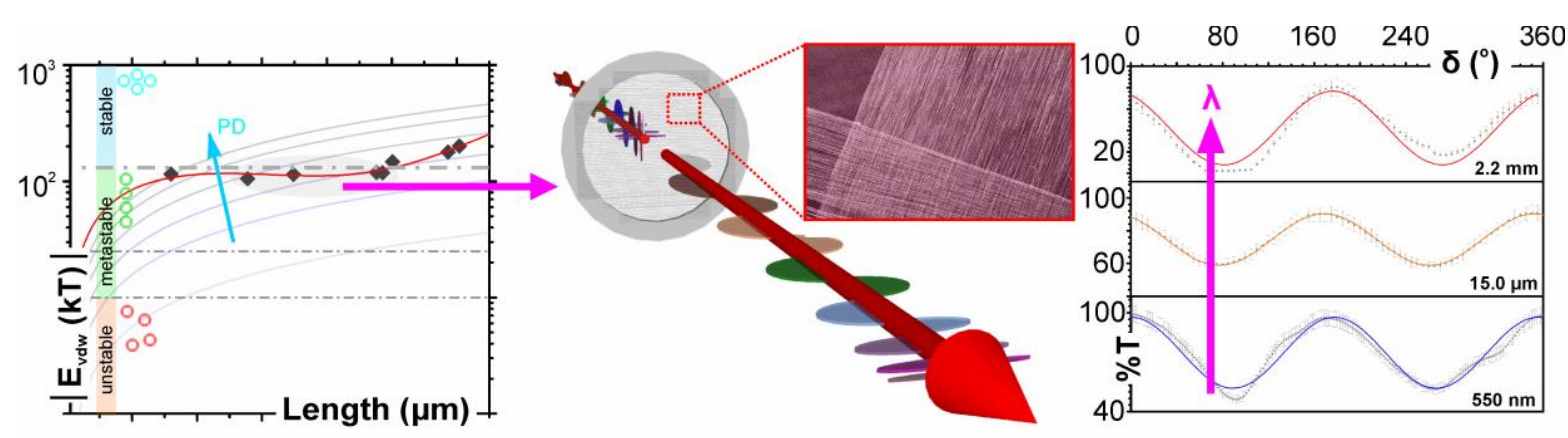
The development of ever broader wavelength $(\lambda)$ light sources is, at present, unmatched by the availability of equally broadband polarisers. Despite the wide catalogue of polarising media available, no single technology offers broadband operation. Conventional polarising media, based on wavelength-comparable anisotropic media or bulk crystals, have well defined operational wavelength ranges determined by a combination of their intrinsic material properties and the necessary fabrication protocols. Most are characterised by a limited normalised bandwidth, $\beta$, of $\sim 4.5$, where $\beta=\log \left(\langle\eta\rangle \lambda_{\max } / \lambda_{\min }\right)$ and $\langle\eta\rangle$ is the mean extinction ratio. Ordered ensembles of multi-walled carbon nanotubes (MWCNTs) are a promising candidate for high performance optical polarising media due to their sub-wavelength scale, near flat absorption, absence of van Hove singularities, and quasi-metallic band structure ${ }^{[11]}$. Here, we investigate the mechanical stability of metastable aligned MWCNT membranes which we use to fabricate a wide wavelength range optical polarising media that may be of particular importance for supercontinuum and synchrotron radiation light sources. The membranes form a pseudo-ordered nanoscopic wire-grid polariser showing linear dichromism over an extremely large bandwidth $(\beta>4.8)$ with extinctions ratios of $2.7 \mathrm{~dB}, 2.3 \mathrm{~dB}$ and $6.4 \mathrm{~dB}$ in the visible, mid infra-red and millimetre wave regimes, respectively.

In birefringent strained-polymeric composite polarisers the optical properties of the substrate limits the usable wavelength range due to dispersion, intrinsic losses, and Fabry-Pérot interference ${ }^{[12]}$. At longer wavelengths, typically in the near infra-red, reduced minor transmittance significantly degrades the blocking capacity. At short wavelengths the principal transmission of common sheet polarisers is dominated by strong absorption of the underlying substrate, with the polarisation efficiency depending primarily on the pitch, periodicity, and conductivity of the embedded linear conductors. Polymer sheet and crystal polarisers are typically opaque for $\lambda>2 \mu \mathrm{m}$, whilst single-crystal polarisers can be prohibitively expensive. Indeed, to date, no single polarising media has proven efficient and cost-effective across ultra-wide wavelength ranges. 
The material properties of the constituent wires have a significant effect on the wavelength-dependent effective dielectric characteristics of the grid polariser. To date, metals are most widely used due to their excellent wideband conductivity and the maturity of thin film deposition processes. However, the refractive and absorption indices of metals decreases with decreasing wavelength, limiting the functional bandwidth, whilst readily excited short-range surface plasmon polaritons result in undesirable spectral absorption peaks. In contrast, semiconductors offer high extinction ratios though at the expense of a greatly reduced bandwidth. The various semiconductors have high reflectional losses and are commonly employed at short wavelengths. Evidently, quasi-metallic, near-zero band gap materials, such as the graphitic carbon allotropes are attractive nanostructures on which to base next-generation wire grid polarisers, particularly those with high-aspect ratios, namely the multi-walled carbon nanotubes (MWCNTs). MWCNTs self-assemble, are naturally anisotropic with very large aspect ratios, and are becoming increasingly inexpensive to synthesise. CNTs have high electrical and thermal conductivity as well as one-dimensional charge carrier confinement along their length, the size of which, along with their diameter can be accurately controlled. In ensembles, MWCNTs have a broad, flat optical absorption spectrum ${ }^{[13,14]}$. They offer facile fabrication with the promise of highly parallelised and rapid self-assembly, with a variation in close-packed lateral structure and spacing that precludes plasmonic effects and that is controllable by carefully tailoring the chemical vapour deposition conditions. Macroscopic en masse alignment by mechanical means, exploiting intrinsic inter-tube van der Waals forces, will allow the fabrication of free-standing MWCNT membranes without the requirement of a functionally deleterious host matrix or substrate.

Photonic crystals, based on regular arrays of semi-metallic discrete graphitic elements, are a theoretically predicted source of Bragg Scattering and strong linear dichromism ${ }^{[15]}$. Visible frequency polarisers have been demonstrated ${ }^{[16]}$, in addition to optical nanoantennas ${ }^{[17]}$ and pulsed THz optical media ${ }^{[10,18,19]}$. Yet, all studies hitherto presented considered CNTs supported on costly sapphire substrates, characterised by time domain spectroscopy ${ }^{[20]}$. Optical polarisation using free-standing MWCNT membranes has been presented 
elsewhere, but only across very narrow wavelength ranges and only in the optical window ${ }^{[10,18]}$. To the best of the authors' knowledge, the stability of free-standing MWCNT membranes and direct measurements of the continuous wave $(\mathrm{CW})$ polarisation over an ultra-wide wavelength window has not yet been investigated. Here we present a route to fabricate free-standing MWCNT membranes and investigate their ultrabroadband polarisation potential under CW-irradiation.

MWCNT polarisers were fabricated in a rapid, facile, inexpensive and highly parallel process. Vertically aligned MWCNT forests were grown by thermal chemical vapour deposition (CVD) (Experimental Section and Supplementary Information), as reported in detail elsewhere ${ }^{[21]}$. The MWCNTs were $25.4 \pm 13.3 \mathrm{~nm}$ in diameter with 2-5 graphitic walls (Supplementary Information, Figure S1b). Solid-state extrusion was employed to fabricate the aligned MWCNT membranes ${ }^{[22,23]}$. Figure 1a shows an optical micrograph during an extrusion process, as depicted in the insert of Figure 1b. To produce the membranes, Kapton tape was attached to the edge of the as-grown MWCNT forest, which was subsequently retracted at a rate of $\sim 10^{-2} \mathrm{~ms}^{-}$ ${ }^{1}$. Membranes had a typical linear packing density of $20 \mu \mathrm{m}^{-1}$, were $\sim 5 \mu \mathrm{m}$ thick, and could be drawn to span significant lengths; an as-grown $10 \times 10 \mathrm{~mm}$ sample is capable of producing a ribbon some $300 \mathrm{~m}$ in length. Throughout, no post-synthesis chemical treatments were performed. Polariser densification was achieved by repeatedly overlaying membranes. In the present study only single layer membranes were considered. Figure 1b shows a typical scanning electron micrograph of a crossed polariser formed from two separate single orthogonal extrusions. Analysis of $>400$ Raman spectra confirmed the presence of graphitic MWCNTs with characteristic $I_{D} / I_{G}(0.26 \pm 0.02), I_{2 D} / I_{G}(0.50 \pm 0.17)$, full-width at half-maximum of the Gpeak $\left(\Gamma_{G}=50.4 \pm 10.7 \mathrm{~cm}^{-1}\right)$ and 2D-peak $\left(\Gamma_{2 D}=78.6 \pm 3.6 \mathrm{~cm}^{-1}\right)$. A Breit-Wigner-Fano G-peak fit evidenced an evolving secondary peak centred at $1922 \mathrm{~cm}^{-1}$ suggesting a quasi-metallic band structure, essential for a flat band absorption spectrum (Figure 2a), whilst spatially resolve Raman measurements indicated that the extrusion process did not induce significant crystallographic disorder with the membranes being homogenous over centimetre-length scales. 
Within the membranes the MWCNTs were highly aligned. The Herman's Orientation Factor, $f=-0.25$, where $-0.5 \leq f \leq 1.0$, and $f=-0.5$ for perfect alignment, $f=0$ denotes a random orientation, and $f=1.0$ for MWCNTs perpendicularly aligned to the extrusion direction (Experimental Section). The solid-state extrusion process outperformed other thin film forming techniques, employing equivalent MWCNTs, for the degree of alignment; such as vacuum filtration $(f=0.02)$, mechanical compression $(0.09)$, and direct shearing (-0.20) (Figure 1c and 1d). The high degree of alignment was independently corroborated by polychromatic polarised Raman spectroscopy which showed a mean full-width at half-maximum of a fittedLorentzian distribution of $13.2^{\circ}$ (532 $\mathrm{nm}$ excitation), as similarly reported elsewhere ${ }^{[24]}$ (Figure $\mathbf{2 b}$, Experimental Section).

Though widely studied theoretically ${ }^{[25,26]}$ due to the challenging fabrication very little empirical evidence is available on the detailed van der Waals binding potential between MWCNTs in well-aligned large-area samples. Two key factors contribute to the thermodynamic stability of a given discrete nanostructured configuration; the degree of adhesion mediated by the inter-tube van der Waals (VdW) and the arrangement and associated many body-effects of the constituent MWCNTs. Our membranes exist in a metastable ribbonlike state. The morphology is intermediary to an unstable disordered geometry and stable, close-packed highly ordered rope-like configurations (Figure 3a). Both boundary states present energetically preferential configurations and minimise the surface free energy of the system. The strength of the VdW binding, which dictates the likely morphology adopted, is here controlled through careful variation in the CVD conditions, namely the MWCNT length and packing density.

Figure 3b shows the length dependence of the Raman defectiveness $\left(I_{D} / I_{G}\right)$, packing density $(P D)$, growth rate $(\rho)$, and surface $s p^{3}$ density. The surface $s p^{3}$ phase showed a weak dependence on the MWCNT length ( $<1 \%$ variation), indicating that stability is likely not a consequence of an $s p^{3}$ mediated electrostatic adhesion 
and that Columbic interactions, mediated by polar surface absorbates, do not play a central role in the observed cohesion. However, most notably, the $P D$ did tend to decrease with MWCNT length, saturating at approximately $9 \times 10^{9} \mathrm{~cm}^{-2}$ (for lengths $>300 \mu \mathrm{m}$ ) due to catalyst poisoning and attrition with subsequent growth termination.

In the present study we assume MWCNTs to be rigid rods, ${ }^{[27]}$ where their length is significantly greater than their diameter. In the first instance, given the planar morphology and that the tube-tube interactions become negligible at centre-to-centre distances greater than twice the tube radius, the membrane can thusly be treated as if only the nearest neighbours are interacting. To estimate the magnitude of the inter-MWCNT adhesion potential we first consider the interaction energy per unit length, $\varphi$, between two parallel multi-walled carbon nanotubes of the same diameter, $D$, and separated by a distance, $d$, using a simplified smeared continuum Leonard-Jones formalism for the carbon-carbon potential, first proposed by Girifalco ${ }^{[28]}$. Such analytical functions have proven validity, as detailed elsewhere when describing the cohesive properties of graphitic solids ${ }^{[29,30]}$. In the case of perfectly aligned cylinders, in the far field $(\mathrm{d}>10 \mathrm{~nm})$, the repulsive term dominates with $\varphi$ tending rapidly toward zero. Certainly, in the far field, based on existing models, there is only a weak dependence on the $P D$ and $D$, with the primary adhesion contribution arising from the interacting length. Nevertheless, even for MWCNTs of considerable length the resultant total interaction potential is negligibly small compared to the ambient thermal potential. Evidently, such simple and idealised models cannot explain our observed stability; long-range $\mathrm{VdW}$ mediated interactions alone do not provide a physical basis for the observed adhesion. Indeed, the universal VdW curves for graphitic carbons do not accurately predict our empirical findings based on this idealised model ${ }^{[26]}$. It is unphysical to assume perfectly aligned MWCNTs throughout the membrane. Rather, we find that the interaction potential depends critically on the detailed orientation of the constitute macromolecules. This entropic contribution must therefore be considered in more detail though the inclusion of a morphology and material dependent parameters. The conventional continuum LJ model precludes configuration components, which can 
effectively exploit the near-field, high cohesiveness in a long-range ordered structure ${ }^{[29]}$. The MWCNTs are pseudo-aligned and interact with one another at oblique, but nonetheless, crossed-junctions. These junctions are bound by short-range attractive $\mathrm{VdW}$, an interaction bolstered by the high polarisability of the graphene lattice $^{[27]}$, with a correspondingly potential of the order of $1 \mathrm{meV} / \AA^{[31]}$. This rapidly approaches zero for inter-tube distances $>3 \mathrm{~nm}^{[27,29]}$.

Equation (1) below shows an augmented LJ analytical expression for the VdW interaction potential based on the $P D, D$ and $\tau$;

$$
E_{v d w}=f(\zeta) \cdot g(\gamma, \tau, \chi) \cdot L\left(\frac{-A}{24}\right) \sqrt{\frac{D}{d^{3}}}
$$

Here, $A$ denotes the Hamaker constant $\left(6 \times 10^{-20} \mathrm{~J}\right), L$ is the MWCNT length, $D$ is the MWCNT diameter, and $d$ is the inter-MWCNT pitch within the nascent vertically aligned forest $\left(\sim P D^{-1 / 2}\right.$, following a similar methodology as proposed by ${ }^{[32]}$ ). Equation (1) increases linearly with $L$ and sub-linearly with $P D$. The negative prefactor denotes attraction. The functions $f(\zeta)$ and $g(\gamma, \tau, \chi)$ signify Coulombic and entropic contributions, respectively, which modulate the effective binding, dramatically enhancing the degree of adhesion for a given morphology and degree of graphtisation. Certainly, the greater the degree of disorder, the higher the entropic contribution and the greater the mechanical robustness of the membrane. Such nonidealities in the membrane morphology where independently assessed by high resolution electron microscopy, including the mean MWCNT overlap zone $(\gamma)$, the mean angle of rotation between individual MWCNTs $(\tau), a-C$ and a surface localised $s p^{3}$ phase $(\zeta)$, and MWCNT waviness $(\chi)$. SEM inspection suggested that $d \sim D, \gamma \sim 0.1$, and $\tau \sim 87^{\circ}$. For simplicity, we assume $\gamma \cdot|\sin (\tau)|$ linearly decreases the effective interaction length, thereby increasing the effective number density of crossed MWCNTs. Experiment showed a weak dependence on $\zeta$ (assumed here to be unity). $\chi$ will be considered in further detail in subsequent studies. For the given morphological parameters during a typical extrusion $\left(P D \approx 10^{10}\right.$ $\mathrm{cm}^{-2}, D \approx 25 \mathrm{~nm}$ and $\left.L \approx 500 \mu \mathrm{m}\right), E_{v d w} \sim 3.9 \mathrm{eV}$. Assuming nominally equivalent tubes we plot the variation 
in $E_{v d w}$ as a function of $L$ (Figure 3c) and its relation to the corresponding measured data (grey squares). A good fit is noted, with a recursive least-squares $\left(\mathrm{R}^{2}\right)$ error $>0.93$ (red curve, Figure 3c). Transient stability, with lifetimes of the order of $\leq 1 \mathrm{~s}$, are evident when $E_{v d w} \leq 10 k T$, whilst the established morphology is longlived (hundreds of hours) when $E_{v d w} \geq 25 k T$. Our models suggest that our membranes exist in a metastable state and can be formed when $9 \leq L \leq 495 \mu \mathrm{m}$. At greater lengths alternative, and increasingly stable configurations are favoured, such as nanostructured ropes. Membranes cannot be drawn exterior to this zone. The highlighted grey area indicates the optimal growth conditions of the nascent forest with the white data point on the force plot in the inset of Figure 3c) denoting the typical inter-MWCNT adhesion force under these nominal growth conditions $(9.4 \mathrm{pN})$. Our findings suggest that the MWCNTs have a preference towards rope formation for $P D>1.4 \times 10^{10} \mathrm{~cm}^{-2}$. We stress that higher PDs may indeed reduce membrane stability causing a migration from the metastable membrane state towards more energetically favourable configurations.

We now consider the degree of optical polarisation from our aligned MWCNT membranes when irradiated with CW linearly polarised visible, IR, and THz (vis-CW, IR-CW, THz-CW) sources spanning an excitation window of $400 \mathrm{~nm}-2.5 \mathrm{~mm}$. The incident plane of polarisation was aligned orthogonal to the MWCNTs long-axis and the membranes were rotated from $\delta=0^{\circ}$ to $360^{\circ}\left(\Delta \delta=2.0^{\circ}\right)$, as illustrated in the inset in Figure 4a. Figure 4a shows typical measured transmission, T, spectra as a function of $\delta$ at $2.2 \mathrm{~mm}, 15.0$ $\mu \mathrm{m}$, and $550 \mathrm{~nm}$. All T $(\delta)$ plots clearly reproduce the Malus' law $\left(A+B \cos ^{2} \delta\right)$, where $A$ and $B$ are empirically determined constants which are intimately related the fine features of the membrane. The inserts of Figure 4a depict spatially resolved transmission maps for the vis-CW, and IR-CW cases, highlighting discrete high contrast areas that are not coincident in the two wavelength regimes. A comprehensive transmission contour plot of the optical transmission as a function of wavelength and $\delta$ is given in Figure $\mathbf{4 b}$. 
For excitations $>2 d(\sim 50 \mathrm{~nm})$ Bragg scattering is suppressed. In metallic ensembles, free carriers dominate resulting in diffuse, flat band absorption over a broad bandwidth. This manifests as an homogenous effective medium where $\eta$ is independent of the fine (sub-nm) features of the structured surface but rather depends critically on its dielectric properties ${ }^{[33]}$. Here, the complex refractive and absorption indices were taken from experimental values for graphite and extended into the $\mathrm{mm}$ range using a Drude-Lorentz model (Experimental Section). Transmission simulations were performed using an effective medium MaxwellGarnett approach (Experimental Section). A good agreement between the wavelength dependent empirical (Figure 4b) and theoretical transmission (Figure 4c, d) was noted. The extinction ratio at $\lambda=550 \mathrm{~nm}(25$ $\mathrm{nm}$ diameter, $30 \mathrm{~nm}$ pitch) was 0.46 , which compared favourably to the measured value of 0.48 (error < $4 \%$ ). For small wavelengths both the real and imaginary parts of the dielectric constant increase, indicating greater absorption and a consequent enhancement in $\eta$ due to the dominance of intra-band transitions. Discrepancies between the model and measurements arise due to unavoidable spatial variation in mass density and pitch, as noted in Figure 4c, where $\eta$ has been plotted for pitches of $\pm 1 \sigma( \pm 13.3 \mathrm{~nm})$ of the mean (25.0 nm). The CW-THz measurements show spectral features not predicted in the model, possibly due to Fabry-Perot interference, likely associated with lateral standing waves. Perhaps the most probable explanation of the apparent discrepancies is the non-perfect alignment of the MWCNTs. This may go some way to explain the $<100 \%$ transmission. Indeed, the cumulative measured transmission depends critically on a complex relationship involving the incommensurate walls of the MWCNTs, individual MWCNTs and MWCNT bundles which all contribute to the observed wide flat band spectra; it is this multi-dimensional, pseudo-ordered hierarchical morphology that is critical to the wideband operation.

Our MWCNT-based ultra-broadband polariser afforded extinction ratios of up to $2.7 \mathrm{~dB}$ (vis), $2.3 \mathrm{~dB}$ (MIR) and $6.4 \mathrm{~dB}(\mathrm{THz})$. The degree of polarisation $\left(\mathrm{DOP}=\left(\mathrm{T}_{\max }-\mathrm{T}_{\min }\right) /\left(\mathrm{T}_{\max }+\mathrm{T}_{\min }\right)\right)$ and $\eta$ are a trade off with bandwidth $^{[34]}$; low $\eta$ facilitates increasingly broadband operation. Discontinuities in the mass density manifest as effective vacancies in the given spectral regimes. Increasing the mass density by controllable 
increasing the number of extruded layers or detailed CVD parameters may offer one possible route to increase the extinction coefficient, though this will necessarily decay the bandwidth.

Our nanostructured sub-wavelength polarisers offer unique opportunities at higher energies, where the conductivity of metals is somewhat reduced; graphite has demonstrated modest polarisation of monochromatic soft X-rays by exploiting its natural structural linear dichromism. Our aligned MWCNT membranes present a negligible absorption cross-section under isotropic and 99\% polarised Synchrotron soft $\mathrm{X}$-ray exposure, typically $<1.9 \%(6-15 \mathrm{keV})$. The tenuous nature and comparatively low mass density of the membranes effectively limits the polarisation window to $<2 \mathrm{eV}$. Indeed, the principle upper-limit on the functional wavelength window, notwithstanding less-critical plasmons such as that at $\sim 14 \mathrm{eV}$, is defined by the plasma edge $\left(\omega_{p}\right)$ - which was empirically evaluated to be around $4.6 \mathrm{eV}^{[35]}$. In the practical limit, as the pitch tends toward zero, the upper polarisation of the apparent dense wire grid tends towards that of bulk structured graphites (2.0-7.0 eV), whilst for sparse arrays $(d>>D), \omega_{p}$ tends toward the THz. At $>5 \mathrm{eV}$ the effective degree of alignment, namely the characteristic dimension defined by the wavelength of the impinging light, reduces significantly, and as such so too does $\eta$. The effective optical porosity of the film also tends to increase and local variations in mass density dramatically reduce performance. The highly aligned zones present an anisotropic absorptive media. The observed absorption is mediated via induced dipoles within the MWCNTs stimulating consequent bolometric effects. Significant local heating is implausible due to the extremely high specific heat of the electrons relative to the graphitic carbon lattice, their long residency time, and the low-level of electron-phonon-dominated lattice interactions. This weak thermal coupling stimulates a linearly confined hot electron population. Some thermalization occurs for perpendicular orientations due to the increase in scatter sites resulting in an increased phonon-electron interaction. Though, we stress that these excited electrons will observe fewer potential features in the perpendicular orientation, such as inter-tube scatter sites, resulting in an modest increase in membrane 
conductivity perpendicular to the MWCNT long axis, particularly at higher energies, which reduces the polarisation function.

Non-specific disorder mechanisms, such as carrier density inhomogeneity and macroscopic lattice strain, may induce minor variations in the membranes optical properties relative to the presented model, though ensemble averaging to a large extent smears out such effects to give an effective homogenous medium of relatively well-defined optical constants. Indeed, splitting of the Raman 2D-peak suggests disordered nanocrystalline graphite with the $\Gamma_{2 \mathrm{D}}=40 \pm 5 \mathrm{~cm}^{-1}$ indicating a mean crystal size of around $10 \mathrm{~nm}^{[36,37]}$. These segregated $s p^{2}$ phases inhibit many, otherwise deleterious, electron-photon interactions along the MWCNTs long-axis. Shell-shell interactions broaden van Hove singularities with weakly resonant modes that are largely indistinguishable. Whilst our previous work has evidenced such electronic signatures using THz$\mathrm{TDS}^{[38]}$, significant peak suppression was noted during $\mathrm{CW}$-irradiation, in part due to the absence of states at the Fermi level. UPS and UV-Vis revealed a semi-metallic character and low-temeprature transport studies indicated a bandgap consistent with that of pyrolytic graphite $(\sim 40 \mathrm{meV})$. Both manifest as a uniform, flat band, and a largely featureless extinction spectrum. The incommensurate nature of the composite graphene planes within MWCNTs ensured absorption peak-suppression, suggesting that, in a broadband context at least, MWCNTs are more appropriate than other nanomaterials, such as single-walled carbon nanotubes ${ }^{[39,40]}$ and semiconducting nanowires. Nevertheless, the reported fabrication approach does not outperform metallic nanowires, but does however offer a number of advantages with regards to the extremely simplified fabrication over large areas, that are substrate-free.

Figure $4 \mathbf{b}$ shows notable spectral aberrations which we rationalise by a heuristic interpretation of the transmission spectrum. Though various dissipative mechanisms quench particular electron resonances, some detailed structure remains, potentially due to the non-carbon component residues in the MWCNTs. Woods anomalies in the guise of marginal asymmetric Fano-like resonances are noted in the NIR and are likely 
caused by shed catalyst distributed along the MWCNTs length. A (002) XRD peak verified the presence of graphitic carbon, whilst spatially resolved EDX evidenced O (10.4 at.\%), Si (0.4 at.\%), and Al (0.2 at.\%) homogenously distributed throughout the MWCNT bulk (Figure S2a). TGA suggested a 1.6\% (by mass) catalyst residue (Figure S2b). Trace $\mathrm{Al}$ and $\mathrm{Si}$ remain from the growth substrate. XPS independently confirmed these findings, evidencing 2-3\% natively oxidised Si throughout (Figure S2c). Localized plasmon enhancement from nanoparticles, formed from shed catalyst, may bolster plasmon-phonon coupling, as has previously been proposed for CNT-composites ${ }^{[41]}$. Nevertheless, such effects are largely screened by rapid thermalization through the multiple potential relaxation pathways. Non-normal incidence may facilitate a diffuse form of the Rayleigh conjecture whilst other surface plasmon polariton resonances are observed as sharp drop-offs in $\eta$, particular around $1 \mu \mathrm{m}$. To investigate these observed spectral peaks further, lowtemperature $(15-300 \mathrm{~K})$ transport studies were conducted. Off-axis transport was characterised by inter-tube hopping whilst the on-axis conduction showed combined inter-tube hopping and Mott conduction, with a transition energy of around $4.3 \mathrm{meV}(\sim 0.29 \mathrm{~mm})$, suggesting that the well-defined size distributions of the nanotubes in the membrane; namely the wall-wall spacing, the tube diameter, and tube length may all in part account for some of the observed spectral aberrations. Further transport studies will be presented elsewhere.

Here we have shown that by careful tuning of the CVD processing conditions we can form, via solid-state mechanical extrusion, well aligned free-standing metastable multi-walled carbon nanotube membranes that offer facile and inexpensive fabrication of ultra-broadband polarisers. We have quantified the critical forest parameters, such as MWCNT length and packing density, necessary to achieve membrane metastability and have established that membranes cannot be drawn exterior to this zone. Our CW transmission studies in the visible to $\mathrm{mm}$ regimes demonstrated broadband polarisation with extinction ratios of up to $6.4 \mathrm{~dB}$. The results presented demonstrate the intrinsic broadband polarising potential of ordered ensembles of MWCNTs that may form the basis of a variety of unique supercontinuum devices in future opto-electronic systems. 


\section{Experimental Section}

Throughout error bars represent $\pm 1 \sigma(n=3)$ from the mean.

Multi-walled carbon nanotube chemical vapour deposition: Degenerately phosphorus-doped, 200nm-thermally-oxidised, $n$-type $<100>\mathrm{Si}$ substrates were degreased in an ultrasonicated acetone bath and rinsed with IPA, DI water and dried with ultra-high purity $\mathrm{N}_{2} .10 \pm 1 \mathrm{~nm} \mathrm{Al}_{2} \mathrm{O}_{\mathrm{x}}(99.99 \%)$ was deposited in a custom-built magnetron sputterer and natively oxidised upon exposure to ambient atmosphere. A $1.0 \pm 0.1 \mathrm{~nm}$ Fe (99.95\%) catalyst was then thermally evaporated (Lesker PVD 75 ) at $1.2 \AA / \mathrm{min}$. Catalyst samples were transferred to a commercially available, cold-walled, thermal chemical vapour deposition reactor (Aixtron, Black Magic) turbo-molecular evacuated to a base pressure of $<3 \times 10^{-3}$ mbar. The reactor was prepressurised to $26( \pm 1)$ mbar under $196 \mathrm{sccm} \mathrm{C}_{2} \mathrm{H}_{2}$ feedstock (dissolved, 95.00\%) and $4 \mathrm{sccm}$ in $\mathrm{H}_{2}(99.98 \%)$. The resistively heated graphite stage was heated to $700^{\circ} \mathrm{C}\left(5^{\circ} \mathrm{C} / \mathrm{s}\right)$ stimulating MWCNT growth. Growth began at $\sim 510^{\circ} \mathrm{C}$ and was terminated by evacuating the chamber to $<10^{-2}$ mbar and cooling to room temperature. The growth rate monotonically decreased with increasing feedstock exposure time with an initial growth rate of $\sim 800 \mathrm{~nm} / \mathrm{s}$.

Packing density and $s p^{3}$ determination: The packing density was calculated as per the methodology outlined by Esconjauregui et al. ${ }^{[42]}$ A Sartorius micro-balance with a standard error of $0.01 \mathrm{mg}$ was used. $s p^{3}$ concentrations were determined by Raman spectroscopy (Renishaw InVia) ${ }^{[43]}$ operated at $457 \mathrm{~nm}$ to give a minimal penetration depth $(<10 \mathrm{~nm})$ and a surface sensitive approach to assess the immediate $s p^{3}$ crosssection. The $s p^{3}$ content was independently corroborated by X-ray photoelectron spectroscopy, giving a value of $\sim 18 \%$, consistent with our Raman findings, performed using an hemispherical spectrometer with a $\mathrm{Al} \mathrm{K}{ }_{\alpha}, \mathrm{X}$-ray source $(h \bar{\omega}=1486.5 \mathrm{eV}$, energy resolution $=0.8 \mathrm{eV})$. 
Materials characterisation: The CVD growth atmosphere was measured in-situ using a Hiden Analytical differentially pumped, electrostatic quadrupole mass spectrometer. Species over 80 amu were negligible. A TA Q500 thermo-gravimetric analyser (TGA) assessed MWCNT purity. Electron micrographs were acquired using a Zeiss 50, a FEI Philips XL30 SFEG, and an Hitachi S5500 HR-SEM fitted with an Inlens detector and a spatially resolved energy dispersive X-ray detector operated at $30 \mathrm{kV}$. A high-resolution FEI Tecnai F20 transmission electron microscope was used to extract the diameter and wall count for $>300$ individual MWCNTs corroborated by atomic force microscopy (Agilent 5400) operated in tapping mode. Spatially resolved polychromatic Raman measurements were conducted on a Renishaw InVia spectrometer operated with triplicate accumulations at $457 \mathrm{~nm}, 532 \mathrm{~nm}, 633 \mathrm{~nm}$, and $785 \mathrm{~nm}$ (spectral resolution $\sim 1 \mathrm{~cm}^{-1}$ ) equipped with a piezo stage (min. step $=100 \mathrm{~nm}$ ) and a 100x objective giving a probe diameter of $1.4 \mu \mathrm{m}$. An incident power of $<5 \mathrm{~mW}$ was used throughout. DC transport and transfer electronic measurements were carried out in an turbo-molecular evacuated $\left(<10^{-6} \mathrm{mbar}\right)$ custom-built cryostat $(15-297 \mathrm{~K})$ using two LABVIEW controlled, BLP 1.9 low-pass filter connected, Keithley 6430 sub-FA source-meters in standard two- and four-terminal geometries. Liquid He bath $\left(4^{\circ} \mathrm{K}\right)$ and high temperature measurements $\left(297-1000^{\circ} \mathrm{K}\right)$ were implemented using a similar set-up. Two and four probe measurements were nominally equivalent across the temperature range considered and were independently verified at room temperature using a Jandel four-probe $100 \mu \mathrm{m}$ contact stylus attached to a Keithley 2100.

Alignment quantification: The degree of alignment was quantified using the Herman's orientation factor, $f$, given by;

$$
f=\frac{3<\cos ^{2} \delta>-1}{2}
$$

where, $\mathrm{I}_{\Delta}$ is the intensity at angle $\delta$, and; 


$$
<\cos ^{2} \delta>=\frac{\int_{0}^{\pi / 2} I_{\Delta} \cos ^{2} \delta \sin \delta d \delta}{\int_{0}^{\pi / 2} I_{\Delta} \sin \delta d \delta}
$$

The polarised Raman spectroscopy technique of Hwang et al. ${ }^{[44]}$ and Ago et al. ${ }^{[45]}$ was adopted to independently corroborate the degree of alignment. The intensity of the $\mathrm{D}, \mathrm{G}$, and 2D Raman peaks at an angle, $\delta$, between the MWCNT and polarization axes, is represented by;

$$
I(\delta)=\int_{\Theta-\pi / 2}^{\Theta+\pi / 2} k \zeta(\delta-\phi, \Delta) \cos ^{4} \delta d \delta
$$

where $\mathrm{I}(\delta)$ is the measured intensity, $\delta$ is the angle between the MWCNT axis and the incident polarised excitation, $k$ is the maximum intensity at $\phi=0$ and the function $\zeta(\delta-\phi, \Delta)$ describes the MWCNT orientation distribution. Assuming a Lorentzian distribution, typical for nanorod ensembles ${ }^{[44]}, \zeta(\delta-\phi, \Delta)$ is given by:

$$
\zeta(\delta-\phi, \Delta)=\frac{\Delta / 2 \pi}{(\delta-\phi)^{2}-(\Delta / 2)^{2}}
$$

from which the degree of alignment $(\Delta)$, following a least squares fit, is extracted.

Continuous wave transmission measurements: UV-vis-NIR $(190 \mathrm{~nm} / 6.53 \mathrm{eV}-1100 \mathrm{~nm} / 1.13 \mathrm{eV})$ transmittance measurements were acquired using a dual beam Thermo spectrophotometer (ATI Unicam UV2) fitted with a $2 \mathrm{~nm}$ resolution monochromator, deuterium and tungsten optical sources and a beam width of $3 \mathrm{~mm}$. MIR $(1.5 \mu \mathrm{m} / 830 \mathrm{meV}-25.0 \mu \mathrm{m} / 49.6 \mathrm{meV})$ transmittance spectra and images were acquired over four accumulations using a Krs5 source polarised Perkin-Elmer atmospherically compensated Frontier 400 FT-IR interferometer using a Spotlight Imaging system with $\mathrm{KBr}$ optics with a $0.4 \mathrm{~cm}^{-1}$ resolution and beam diameter of $8.9 \mathrm{~mm}$. Transmission maps were averaged across the full MIR spectral range with a 5 x $5 \mu \mathrm{m}$ pixel resolution. Millimetre wave $(2.10 \mathrm{~mm} / 0.59 \mathrm{meV}-2.55 \mathrm{~mm} / 0.48 \mathrm{meV})$ 
transmittance was measured using a contactless technique ${ }^{[46]}$ employing a 716 Micro-Now Inc. millimetre wave sweep generator and a carcinotron backward wave oscillating travelling-wave vacuum tube $(<0.2$ $\mathrm{mW}$ ), producing a beam cross-section of less than $1 \mathrm{~mm}^{2}$ with a spectral resolution of $1 \mathrm{MHz}$, adjacent to a HP 432A power meter. Two dielectric waveguides, one coupled to the radiation source and the other to a horn and power meter, were manoeuvred with micrometres normal to the suspended MWCNTs.

All transmission experiments were conducted in continuous wave mode, in air at STP, with a source detector separation of $\sim 20 \mathrm{~mm}$ and the source polarizer fixed and the specimen rotated. Specimen rotation was monitored using a manually indexed optical mount $\left( \pm 1.0^{\circ}\right)$, and a computer controlled stepper motor $\left( \pm 1.5^{\circ}\right)$. $2.0^{\circ}$ increments were used throughout. Transmission measurements were normalised to $100 \%$ transmission.

Numerical transmission simulations: Transmittance models were based on a Maxwell-Garnett effective media approach and verified by full numerical simulations. Due to their inherent one-dimensional nature, MWCNTs present an anisotropic effective dielectric response. For a planar wire array, where the diameter of the MWCNT, considered here as simple cylinders, is much smaller than the wavelength of the incident radiation, the composite structure behaves as a homogeneous uniaxial anisotropic sub-wavelength composite. The frequency $(\omega)$ dependent complex index is $N(\omega)=n(\omega)+i k(\omega)$, where $n$ is the refractive index and $k$ is the absorption index. The effective refractive indices of an array of wires of complex refractive index $N_{2}(\omega)$ embedded in a host medium of index, $N_{1}(\omega)$, is given by:

$$
\begin{gathered}
N_{p}^{2}=\xi N_{2}^{2}+(1-\xi) N_{1}^{2} \\
N_{s}^{2}=N_{1}^{2}+\left(\frac{N_{1}^{2} \xi\left(N_{2}^{2}-N_{1}^{2}\right)}{N_{1}^{2}+(1-\xi)\left(N_{2}^{2}+N_{1}^{2}\right)}\right)
\end{gathered}
$$

where $\xi$ denotes a filling factor ${ }^{[47-49]}$ and the subscripts " $p$ " and " $s$ " indicate incident polarisation perpendicular (off-axis) and parallel (on-axis) to the MWCNTs long axis, respectively ${ }^{[50]}$. 
Due to inherent measurement difficulties, to-date the broadband dielectric properties of individual MWCNTs have not been empirically assessed to any great degree. Nor have any substantial theoretical works been developed to this end. Previous studies have instead based their descriptions on the optical constants of bulk graphite and few-layer layer graphene. The former approach was adopted, for example, in an analysis of dense carpets of vertically aligned MWCNTs ${ }^{[51]}$, whilst the latter approach was utilised in ${ }^{[15]}$. Specification of the optical properties of carbon nanotubes is of key importance across a range of applications - data based on the analysis of graphene by ${ }^{[52]}$ were successfully used in understanding the elastic, polarisation-sensitive, optical and inelastic, surface-enhanced Raman response of short, vertically aligned MWCNTs nanotubes ${ }^{[53]}$. In addition, there have been a number of rigorous theoretical analyses of the optical response of carbon nanotubes such as those by Lin $e t a l^{[54]}$ and Shuba et $a l .{ }^{[5]}$. Here, however, we have opted to derive a $\omega$ dependent continuum of the transverse optical constants of graphite from $10^{-4}$ to $10 \mathrm{eV}$, adapted from ${ }^{[56]}$, in order to approximate the broadband optical properties of the constituent MWCNTs. Indeed, linearly and spherically 'rolled' graphite have been employed in a similar way in the past to determine the electron energy loss spectra of MWCNTs and multi-shell fullerenes ${ }^{[57]}$. Data was fitted to a Drude-Lorentzian model so as to extend the predictions into the $\mathrm{mm}$ range where the dielectric function is dominated by the free electron or intra-band response. The dielectric constant of the MWCNT array is thusly given by;

$$
\varepsilon_{s}(\omega)=\left\{1-\frac{\omega_{p l}^{2}}{\omega^{2}+i \omega / \tau}+\sum_{m=1}^{M} \frac{\sigma_{m}^{2}}{\omega_{m}^{2}-\omega^{2}-i \omega \gamma_{m}}\right\}=\varepsilon_{1}(\omega)+i \varepsilon_{2}(\omega)
$$

where the refractive indices $n(\omega)$ and $k(\omega)$ can be determined from;

$$
\begin{aligned}
& n(\omega)=\left\{\frac{1}{2}\left(\varepsilon_{1}(\omega)+\sqrt{\varepsilon_{1}^{2}(\omega)+\varepsilon_{2}^{2}(\omega)}\right\}^{1 / 2}\right. \\
& k(\omega)=\left\{\frac{1}{2}\left(-\varepsilon_{1}(\omega)+\sqrt{\varepsilon_{1}^{2}(\omega)+\varepsilon_{2}^{2}(\omega)}\right\}^{1 / 2}\right.
\end{aligned}
$$

The first term of equation (7) denotes the Drude free-electron metallic intra-band components and the second, the Lorentz oscillatory term, accounts for semiconducting inter-band transitions. $\omega_{p l}$ is the free- 
electron plasma frequency $(4.43 \mathrm{eV}), \tau$ is the free-electron relaxation time $\left(2.32 \mathrm{eV}^{-1}\right), \hbar \omega_{m}$ is the inter-band transition energies, $\sigma_{m}$ is the oscillator strength, and $\gamma_{m}$ is the damping constant. The free electron constants used were from ${ }^{[58]}$ and $m$ was taken as the number of damped oscillators. Such approaches have been experimentally validated from 1-40 eV elsewhere, and were found to be consistent with Continuity and Kramers-Konig $^{[15,59]}$.

Returning to the effective medium models, the host medium was air $\left(\left\langle n_{l}\right\rangle=1\right)$. MWCNTs were modelled as solid entities formed from concentrically nested shells, all way to their centres. The lack of an internal cavity gave negligible loss in model validity; both hollow and solid core systems were modelled, with the latter offering much more rapid computing. Effective media models were corroborated using full-wave numerical simulations. The electromagnetic response was simulated under periodic boundary conditions using a commercial finite element method package with an adaptive meshing routine (COMSOL v3.5a). Surfaces were approximated by an infinite periodic array of cylindrical quasi-metallic cylinders embedded in a dielectric. The optical transmission was calculated for plane-wave polarized radiation at normal incidence for wavelengths between $300 \mathrm{~nm}$ and $4.0 \mathrm{~mm}$. 
Corresponding Author

*Phone : : +44-1223-748304

*Email : mtc35@cam.ac.uk

\section{Supporting Information}

Supporting Information is available from the Wiley Online Library or from the author.

\section{Acknowledgements}

This work was supported by the Isaac Newton Trust, Trinity College Cambridge University. The authors' are grateful to the Cavendish Laboratory, Cambridge University for the kind use of their Raman spectroscopy and electron microscopy facilities. The authors' thank R. Spragg and K. Palmer (Perkin Elmer) for their assistance in acquiring infra-red transmission measurements. We also thank S. G. Shivareddy for TGA, R. Xie for TEM, and C. Cepek for XPS measurements. We acknowledge D. G. Hasko for fruitful discussions. M. T. Cole thanks the Winston Churchill Memorial Trust and the Oppenheimer Research Fellowship, Cambridge University, for generous financial support.

Received: ((will be filled in by the editorial staff))

Revised: ((will be filled in by the editorial staff)) Published online: ((will be filled in by the editorial staff)) 


\section{References}

[1] M. T. Cole, C. Li, Y. Zhang, S. G. Shivareddy, J. S. Barnard, W. Lei, B. Wang, D. Pribat, G. A. J. Amaratunga, W. I. Milne, ACS Nano 2012, 6, 3236-3242.

[2] Teo, K. B. In Plasma Enhanced Chemical Vapor Deposited Carbon Nanotubes for Field Emission Applications, Materials Research Society, 2001.

[3] H. W. Zhu, C. L. Xu, D. H. Wu, B. Q. Wei, R. Vajtai, P. M. Ajayan, Science 2002, 296, 884-886.

[4] M. Zhang, S. Fang, A. A. Zakhidov, S. B. Lee, A. E. Aliev, C. D. Williams, K. R. Atkinson, R. H. Baughman, Science 2005, 309, 1215-1219.

[5] J. E. Jang, S. N. Cha, Y. J. Choi, D. J. Kang, T. P. Butler, D. G. Hasko, J. E. Jung, J. M. Kim, A. J. AmaratungaGehan, Nat. Nano. 2008, 3, 26-30.

[6] H. Ago, K. Petritsch, M. S. P. Shaffer, A. H. Windle, R. H. Friend, Adv. Mater. 1999, 11, 1281-1285.

[7] P. Calvert, Nature 1999, 399, 210-211.

[8] W. X. Chen, J. Y. Lee, Z. Liu, Carbon 2003, 41, 959-966.

[9] B. G. Kang, Y. J. Lim, K.-U. Jeong, K. Lee, Y. H. Lee, S. H. Lee, Nanotech. 2010, 21, 1-5.

[10] J. Kyoung, E. Y. Jang, M. D. Lima, H.-R. Park, R. O. Robles, X. Lepró, Y. H. Kim, R. H. Baughman, D.-S. Kim, Nano Lett. 2011, 11, 4227-4231.

[11] K. Fu, O. Yildiz, H. Bhanushali, Y. Wang, K. Stano, L. Xue, X. Zhang, P. D. Bradford, Adv. Mater. 2013, 25, 51095114.

[12] Q. Gan, H. Hu, H. Xu, K. Liu, S. Jiang, A. N. Cartwright, leee Photonics Journal 2011, 3, 1083-1092.

[13] J. Yu, N. Grossiord, C. E. Koning, J. Loos, Carbon 2007, 45, 618-623.

[14] Z. Wang, Q. Liu, H. Zhu, H. Liu, Y. Chen, M. Yang, Carbon 2007, 45, 285-292.

[15] E. Lidorikis, A. C. Ferrari, ACS Nano 2009, 3, 1238-1248.

[16] S. Shoji, H. Suzuki, R. P. Zaccaria, Z. Sekkat, S. Kawata, Phys. Rev. B 2008, 77, 1534071-1534074.

[17] H. Ajiki, T. Ando, Physica B 1994, 201, 349-352.

[18] L. Ren, C. L. Pint, L. G. Booshenri, W. D. Rice, X. F. Wang, D. J. Hilton, K. Takeya, I. Kawayama, M. Tonouchi, R. H. Hauge, J. Kono, Nano Lett. 2009, 9, 2610-2613.

[19] T.-I. Jeon, K.-J. Kim, C. Kang, I. H. Maeng, J.-H. Son, K. H. An, J. Y. Lee, Y. H. Lee, J. Appl. Phys. 2004, 95, 57365740.

[20] E. P. J. Parrott, J. A. Zeitler, J. McGregor, S.-P. Oei, H. E. Unalan, W. I. Milne, J.-P. Tessonnier, D. S. Su, R. Schlögl, L. F. Gladden, Adv. Mater. 2009, 21, 3953-3957.

[21] M. T. Cole, P. Hiralal, K. Ying, C. Li, Y. Zhang, K. B. K. Teo, A. C. Ferrari, W. I. Milne, J. Nanomat. 2012, 8.

[22] L. Xiao, Z. Chen, C. Feng, L. Liu, Z. Q. Bai, Y. Wang, L. Qian, Y. Y. Zhang, Q. Q. Li, K. L. Jiang, S. S. Fan, Nano Lett. 2008, 8, 4539-4545.

[23] L. Kai, S. Yinghui, C. Lei, F. Chen, F. Xiaofeng, J. Kaili, Z. Yonggang, F. Shoushan, Nano Lett. 2008, 8, 700-705.

[24] T. D. Fornes, J. W. Baur, Y. Sabba, E. L. Thomas, Polymer 2006, 47, 1704-1714.

[25] A. I. Zhbanov, E. G. Pogorelov, Y.-C. Chang, ACS Nano 2010, 4, 5937-5945.

[26] E. G. Pogorelov, A. I. Zhbanov, Y.-C. Chang, S. Yang, Langmuir 2011, 28, 1276-1282.

[27] S. V. Ahir, E. M. Terentjev, S. X. Lu, B. Panchapakesan, Phys. Rev. B 2007, 76, 165437.

[28] L. A. Girifalco, The Journal of Physical Chemistry 1992, 96, 858-861.

[29] L. A. Girifalco, M. Hodak, R. S. Lee, Phys. Rev. B 2000, 62, 13104-13110.

[30] J. Kleis, P. Hyldgaard, E. Schröder, Computational Materials Science 2005, 33, 192-199.

[31] E. Schroder, P. Hyldgaard, Mater. Sci. Eng. C-Biomimetic Supramol. Syst. 2003, 23, 721-725.

[32] S. Esconjauregui, R. Xie, M. Fouquet, R. Cartwright, D. Hardeman, J. Yang, J. Robertson, J. Appl. Phys. 2013, $113,-$.

[33] J. B. Pendry, L. Martín-Moreno, F. J. Garcia-Vidal, Science 2004, 305, 847-848.

[34] F. Hipolito, A. J. Chaves, R. M. Ribeiro, M. I. Vasilevskiy, V. M. Pereira, N. M. R. Peres, Phys. Rev. B 2012, 86, 115430. 
[35] J. B. Pendry, A. J. Holden, D. J. Robbins, W. J. Stewar, J. Phys. Cond. Matt. 1998, 10.

[36] L. G. Canado, K. Takai, T. Enoki, M. Endo, Y. A. Kim, H. Mizusaki, A. Jorio, L. N. Coelho, R. Magalhães-Paniago, M. A. Pimenta, Appl. Phys. Lett. 2006, 88, 1631061-1631063.

[37] L. G. Cançado, A. Jorio, M. A. Pimenta, Phys. Rev. B 2007, 76, 0643041-0643047.

[38] G. Galimberti, S. Ponzoni, A. Cartella, M. T. Cole, S. Hofmann, C. Cepek, G. Ferrini, S. Pagliara, Carbon.

[39] M. F. Islam, D. E. Milkie, C. L. Kane, A. G. Yodh, J. M. Kikkawa, Phys. Rev. Lett. 2004, 93, 037404.

[40] S. Maruyama, Y. Murakami, E. Einarsson, T. Edamura, Carbon 2005, 43, 2664-76.

[41] A. E. Nikolaenko, F. De Angelis, S. A. Boden, N. Papasimakis, P. Ashburn, E. Di Fabrizio, N. I. Zheludev, Phys. Rev. Lett. 2010, 104.

[42] S. Esconjauregui, M. Fouquet, B. C. Bayer, C. Ducati, J. Robertson, physica status solidi (b) 2011, 248, 25282531.

[43] A. C. Ferrari, J. Robertson, Physical Review B - Condensed Matter and Materials Physics 2000, 61, 1409514107.

[44] J. Hwang, H. H. Gommans, A. Ugawa, H. Tashiro, R. Haggenmueller, K. I. Winey, J. E. Fischer, D. B. Tanner, A. G. Rinzler, Phys. Rev. B 2000, 62, 13310-13313.

[45] H. Ago, N. Uehara, K.-i. Ikeda, R. Ohdo, K. Nakamura, M. Tsuji, Chem. Phys. Lett. 2006, 421, 399-403.

[46] R. J. Collier, D. G. Hasko, J. Appl. Phys. 2002, 91, 2547-2549.

[47] J. C. Maxwell, A Treatise on electricity and Magnetism. Cambridge, 1873.

[48] L. Rayleigh, Philos. Mag. 1892, 34.

[49] P. Yeh, SPIE 1981, 307.

[50] C. F. Bohren, D. R. Huffman, Wiley-VCH: 2004.

[51] T. De Los Arcos, P. Oelhafen, D. Mathys, Nanotech. 2007, 18, 1-5.

[52] M. Bruna, S. Borini, Appl. Phys. Lett. 2009, 94, 031901.

[53] P. Dawson, J. A. Duenas, M. G. Boyle, M. D. Doherty, S. E. J. Bell, A. M. Kern, O. J. F. Martin, A. S. Teh, K. B. K. Teo, W. I. Milne, Nano Lett. 2011, 11, 365-371.

[54] M. F. Lin, K. W. K. Shung, Phys. Rev. B 1994, 50, 17744-17747.

[55] M. V. Shuba, G. Y. Slepyan, S. A. Maksimenko, C. Thomsen, A. Lakhtakia, Phys. Rev. B 2009, 79, 155403.

[56] E. D. Palik, Hanbook of Optical Constants of Solids, Vol. II. 1 ed.; Academic Press Ltd.: San Diego, 1991.

[57] E. Najafi, D. H. Cruz, M. Obst, A. P. Hitchcock, B. Douhard, J.-J. Pireaux, A. Felten, Small 2008, 4, 2279-2285.

[58] H. R. Philipp, Phys. Rev. B 1977, 16, 2896-2900.

[59] A. B. Djurisic, E. H. Li, J. Appl. Phys. 1999, 85, 7404-7410. 


\section{Figures}

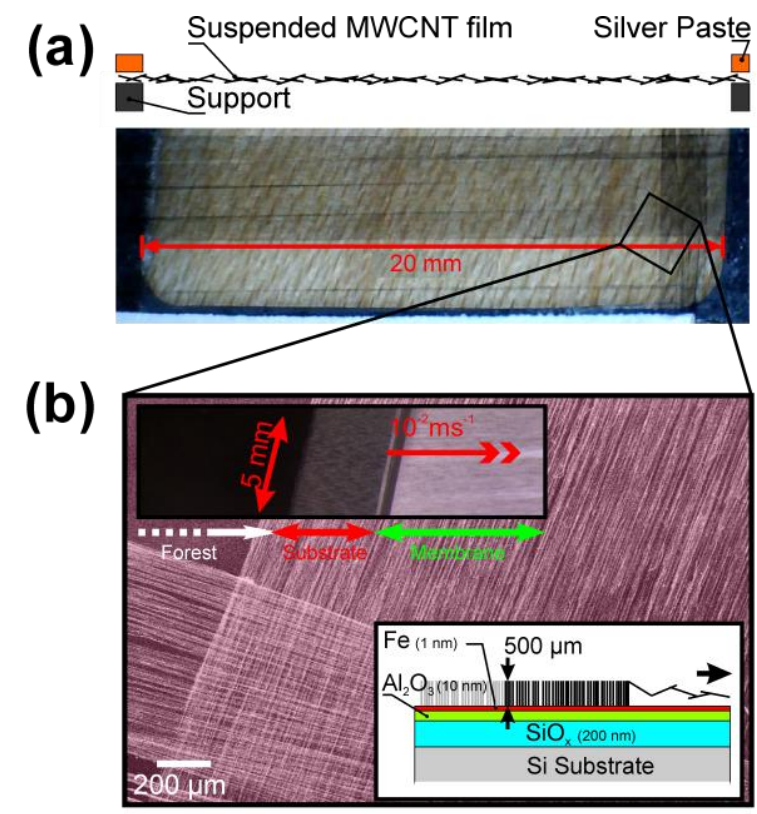

(c)
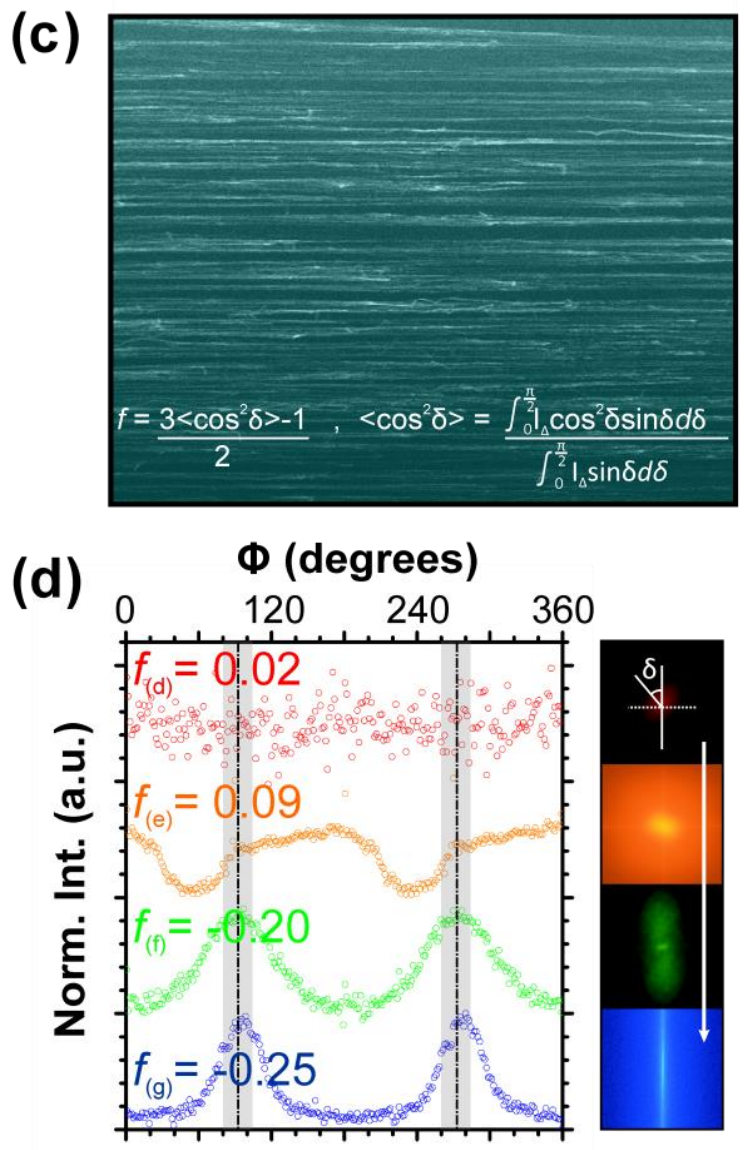

Figure 1. (Colour online). (a) Schematic of a typical polariser and an optical micrograph of a suspended hierarchical polariser following mechnical extrusion from a vertically aligned MWCNT forest synthesised by thermal chemical vapor deposition. Note the opaque crossed- 
region. Inset: Cross-section schematic of the $\mathrm{Al}_{2} \mathrm{O}_{\mathrm{x}} / \mathrm{Fe}(10 / 1 \mathrm{~nm})$ catalysed MWCNTs on a $\mathrm{Si} / \mathrm{SiO}_{2}(200 \mathrm{~nm})$ substrate. (b) Optical micrograph of a $5 \mathrm{~mm}$ wide horizontally aligned MWCNT membrane extruded at $10^{-2} \mathrm{~ms}^{-1}$. A scanning electron micrograph (false coloured) of a crossed MWCNT membrane ultra-broadband CW polariser (Scale bar: $200 \mu \mathrm{m})$. Inset: Schematic depicting the MWCNT growth substrate profile. (c) An example scanning electron micrograph of an aligned, free-standing MWCNT membrane (Scale bar: $10 \mu \mathrm{m}$ ). (d) Hermans Orientation Factor comparing equivalent source MWCNTs formed into thin films by; $f_{(d)}$ mixed cellulose ester membrane vacuum filtration (red), $f_{(e)}$ mechanical compression $\left(500 \mathrm{~g} / \mathrm{cm}^{2}\right)$ (orange), $f_{(f)}$ mechanical rolling (green) $\left(\sim 7 \mathrm{~N}\right.$ at $\left.10^{-2} \mathrm{~m} / \mathrm{s}\right)$, and $f_{(g)}$ mechanical extrusion (blue) [this work]. Inset: FFTs of typical SEM micrographs, for the different alignment techniques stated, showing a progressive increase in alignment. 
(a)

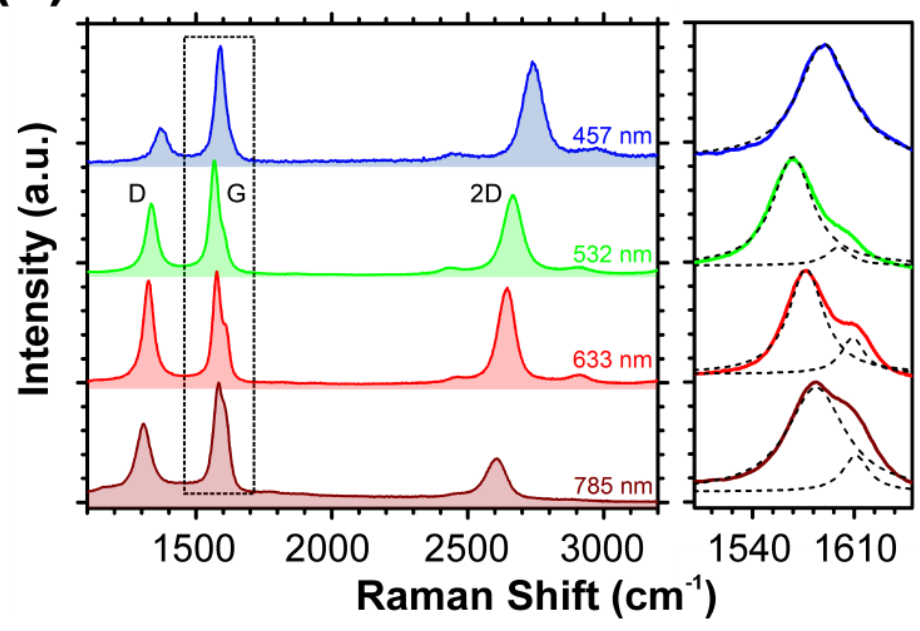

(b)

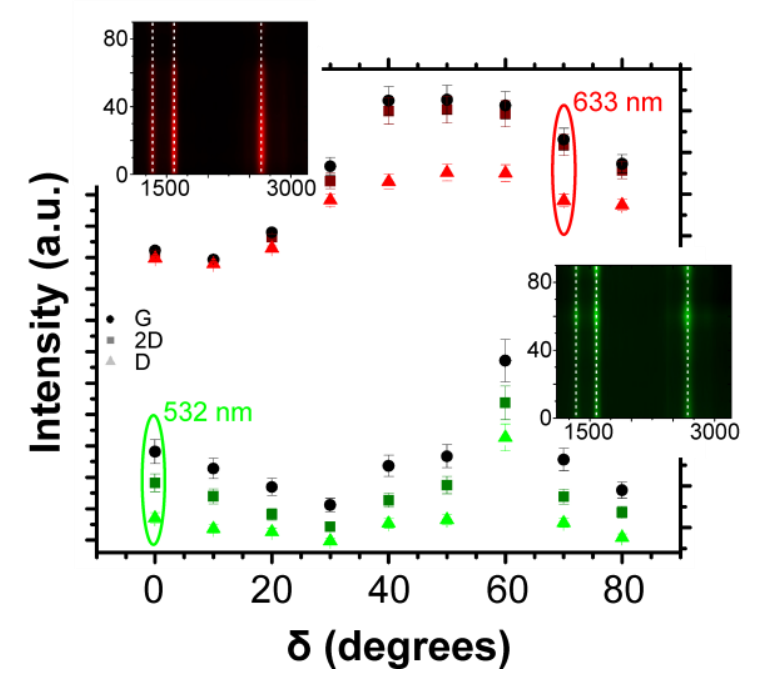

Figure 2. (Colour online). (a) Typical Raman spectra at 457, 532, 633, and $785 \mathrm{~nm}$ unpolarised Raman spectra of an aligned MWCNT sample evidencing strong G-splitting with increasing wavelength. Breit-Wigner-Fano G-peak fits show an evolving secondary peak centred at 1922 $\mathrm{cm}^{-1}$ due to a quasi-metallic band structure. Equivalent orientations were used in each scan. (b) Polarised Raman spectroscopy for $0^{\circ} \leq \delta \leq 80^{\circ}$ at $633 \mathrm{~nm}$ and $532 \mathrm{~nm}$ excitation showing the angle-resolved D (1339 $\left.\mathrm{cm}^{-1}\right), \mathrm{G}\left(1576 \mathrm{~cm}^{-1}\right)$ and 2D (2673 $\left.\mathrm{cm}^{-1}\right)$ Raman peaks. Insets: Fullspectrum angle-resolved $633 \mathrm{~nm}$ (red) and $532 \mathrm{~nm}$ (green) maps. 
(a)

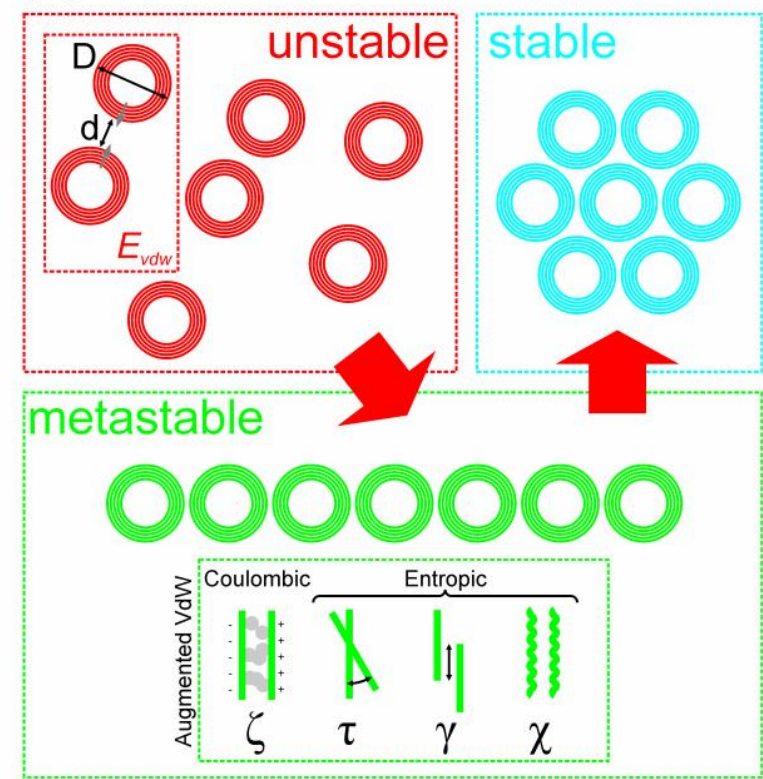

(b)

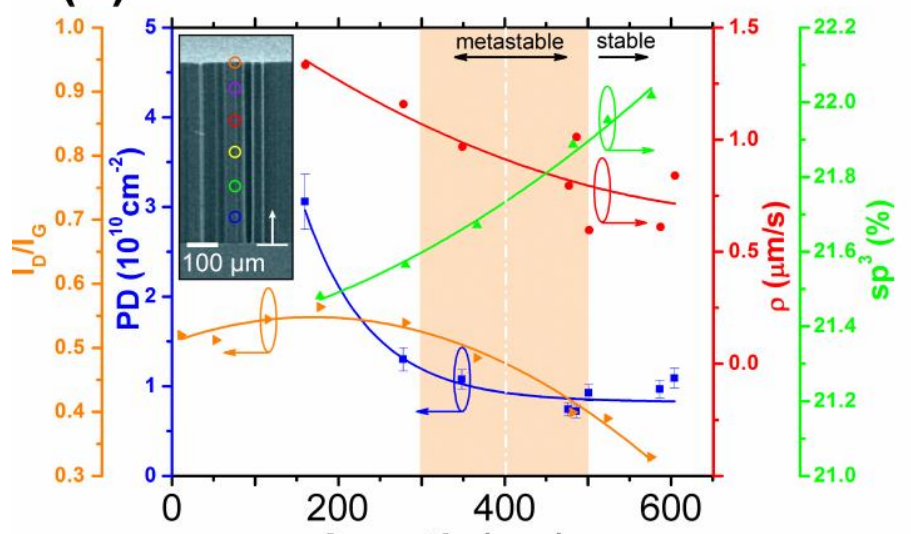

(c)

Length $(\mu \mathrm{m})$

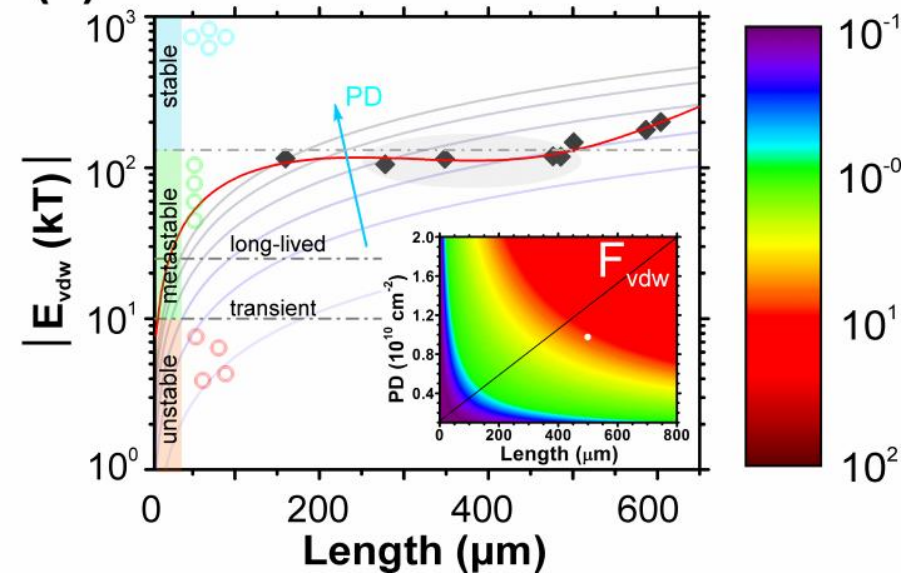

Figure 3. (Colour online). (a) Depiction of unstable, metastable and stable morphologies, and their dependence on the spatial distribution of the MWCNTs and the inter-MWCNT van der waals binding, $E_{v d w}$ which is a function of the MWCNT diameter $(D)$, MWCNT separation $(d)$, 
in addition to Coulombic contributions, principally $s p^{3}$ phases ( $\zeta$ ), and entropic contributions, including MWCNT overlap zone $(\gamma)$, the rotation between individual MWCNTs $(\tau)$, and the MWCNT waviness $(\chi)$. (b) Measured length dependence in the MWCNT; defectiveness $\left(I_{D} / I_{G}\right)$, packing density $(P D)$, growth rate $(\rho)$, and surface $s p^{3}$ content. Inset: Typical scanning electron micrograph depicting the approximate locations of $s p^{3}$ evaluation (Scale bar: $\left.100 \mu \mathrm{m}\right)$. (c) Theoretical (red curve) and measured (grey diamonds) variation in the inter-MWCNT van der Waals interaction energy $\left(E_{v d w}\right)$ for idealised aligned membrane formed from rod-like carbon macromolecules, as a function of packing density and MWCNT length. The shaded grey are signifies the approximate optimal conditions for membrane formation. $k T$ denotes the thermal energy and is $4.11 \times 10^{-21} \mathrm{~J}$ at STP. Inset: Length and packing density dependence of the interMWCNT van der Waals force $\left(F_{V d W}\right)$. Units of $\mathrm{pN}$. The white point highlights typical growth conditions. 

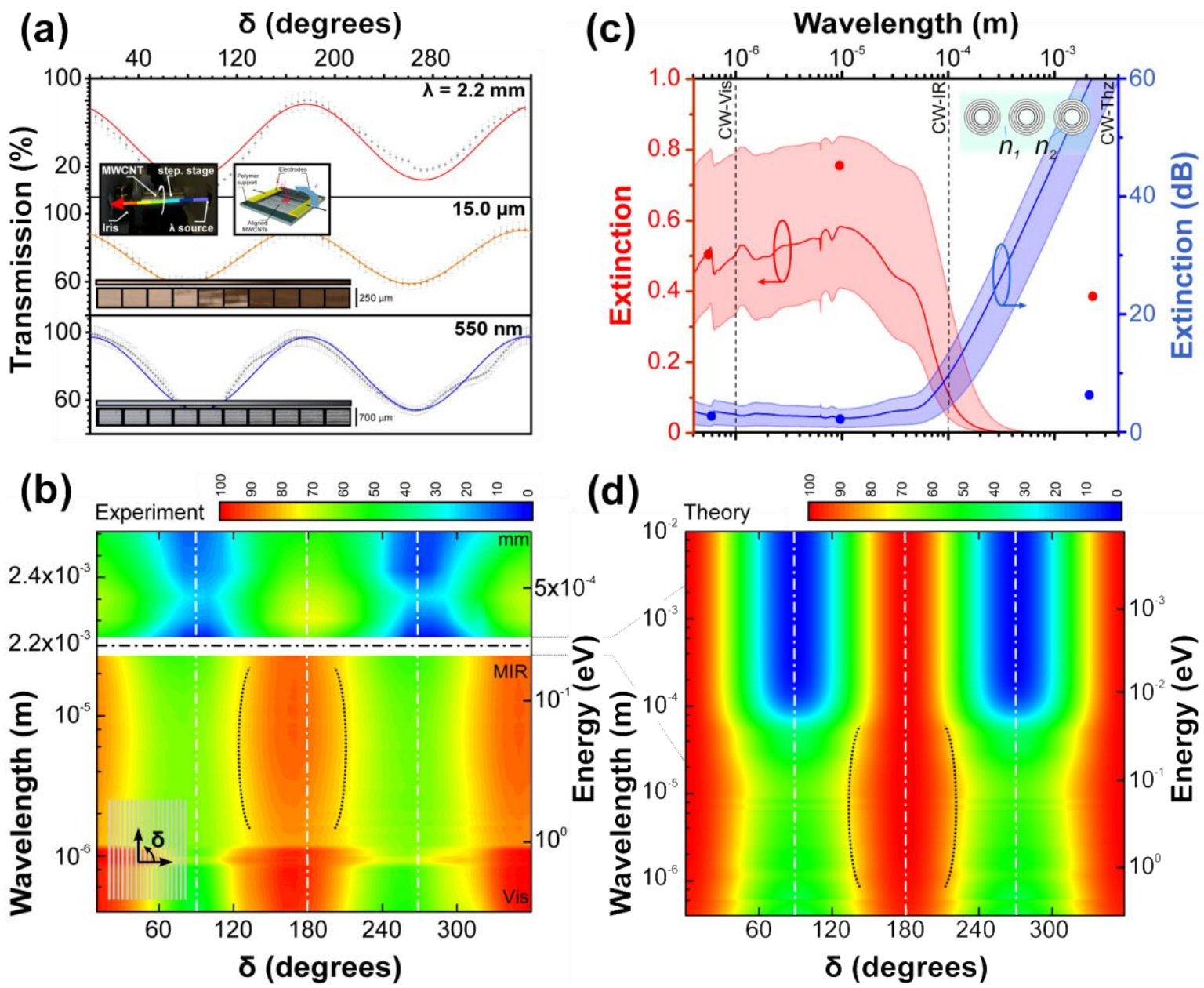

Figure 4. (Colour online). (a) Example measured continuous wave transmission (\%) as a function of azimuthal angle $(\delta)$ at $550 \mathrm{~nm}$ (Vis), $15 \mu \mathrm{m}$ (MIR), and $2.2 \mathrm{~mm}(\mathrm{~mm})$ with corresponding $\cos ^{2} \delta$ fits. $0^{\circ} / 90^{\circ}$ denotes s/p-polarised incidence. Inset: Experimental setup. The incident beam is linearly polarised perpendicular to the MWCNTs long-axis $\left(0^{\circ}\right)$ with the membrane rotated from $\delta=0^{\circ}-360^{\circ}$. Spatially resolved transmission maps acquired at $10^{\circ}$ increments $-0^{\circ}$ (transparent), $90^{\circ}$ (opaque) for integrated intensities across the NIR (1.5 - 25.0 $\mu \mathrm{m}$, Scale bar: $250 \mu \mathrm{m})$ and optical (300 - $900 \mathrm{~nm}$, Scale bar: $700 \mu \mathrm{m})$ spectrums. (b) Measured ultra-broadband transmission as a function of azimuth and wavelength. A reduction in transmission of $>50 \%$ transmission is noted for $400 \mathrm{~nm}-2.5 \mathrm{~mm}$. (c) Simulated broadband extinction ratio $\left(=T_{p} / T_{s}\right)$ in standard units and $\mathrm{dB}$. Dots denote measurements. Errors stated as \pm 1 s.d $(\mathrm{n}=3)$ in nanotube diameter $(25.0 \pm 13.3 \mathrm{~nm})$ a $25 \mathrm{~nm}$ pitch. (d) Simulated transmission as a function of azimuth and wavelength. 


\section{Supplementary Information}

(a)

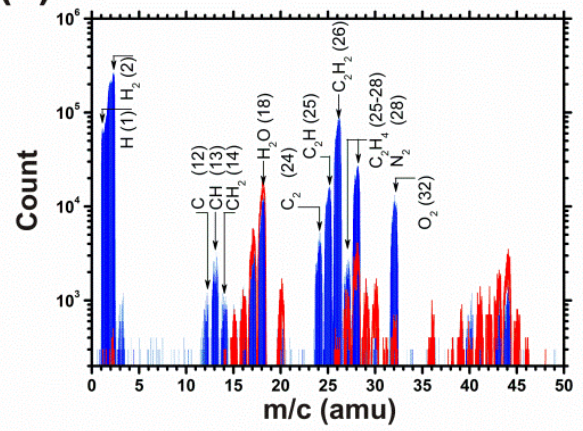

(b)

Pitch (nm)

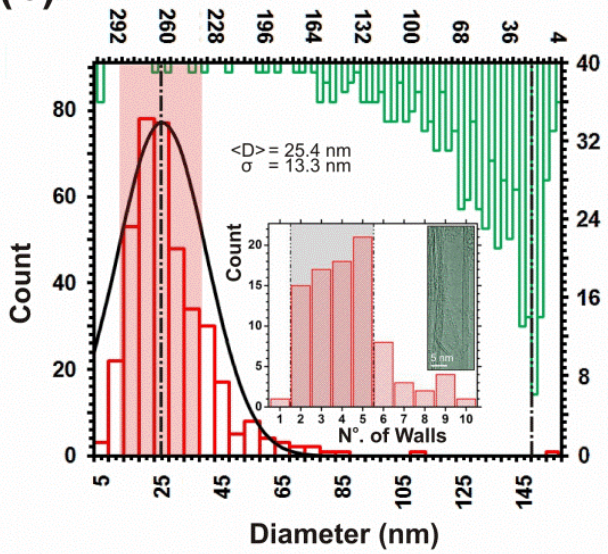

(c)

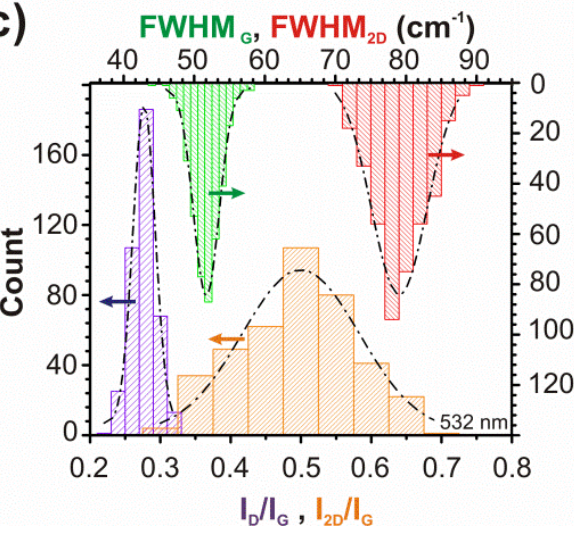

Figure S1. (Colour online). (a) In-situ mass spectra of the background atmosphere (red), and growth atmosphere (blue) during chemical vapour deposition $\left(\mathrm{C}_{2} \mathrm{H}_{2}(4 \mathrm{sccm}): \mathrm{H}_{2}(196 \mathrm{sccm})\right)$ showing prominent $\mathrm{H}(1 \mathrm{amu}), \mathrm{H}_{2}(2 \mathrm{amu}), \mathrm{N}_{2}(28 \mathrm{amu}), \mathrm{O}_{2}(32 \mathrm{amu})$, and $\mathrm{C}_{\mathrm{x}} \mathrm{H}_{\mathrm{y}}(1<x<2$, $0<y<4)(12-28 \mathrm{amu})$ signatures. (b) Normal distribution in MWCNT diameter $(25.4 \pm 13.3 \mathrm{~nm})$, and inter-MWCNT pitch $(25 \mathrm{~nm})$. The inset shows an example high-resolution transmission electron micrograph of an as-synthesised MWCNT (Scale bar: $5 \mathrm{~nm}$ ) and the wall count distribution $(2-5 \mathrm{~nm})$. (c) Statistical variation over 400 scans, at $532 \mathrm{~nm}$ excitation, showing Gaussian fits to the $\mathrm{I}_{\mathrm{D}} / \mathrm{I}_{\mathrm{G}}(0.26 \pm 0.02), \mathrm{I}_{2 \mathrm{D}} / \mathrm{I}_{\mathrm{G}}(0.50 \pm 0.17)$, the full-width at half-maximum of the G-peak $\left(\Gamma_{\mathrm{G}}=50.4 \pm 10.7 \mathrm{~cm}^{-1}\right)$, and 2D-peak $\left(\Gamma_{2 \mathrm{D}}=78.6 \pm 3.6 \mathrm{~cm}^{-1}\right)$. Normal distribution fits shown. 
(a)

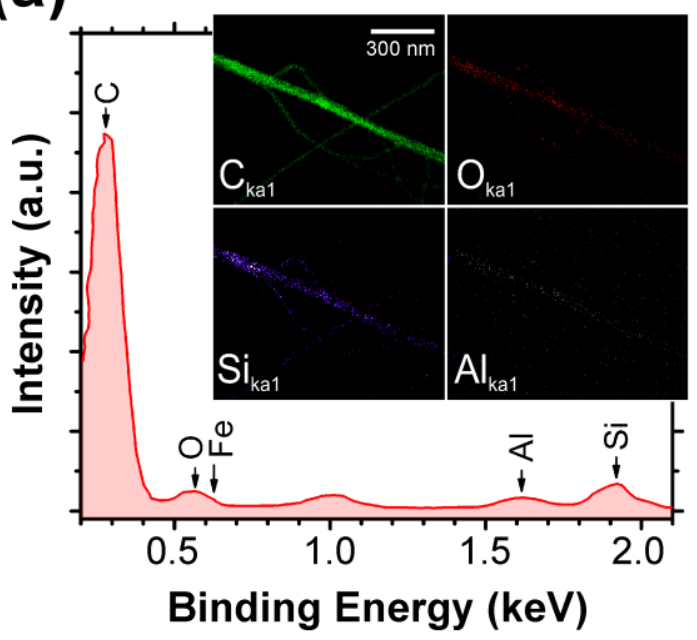

(b)

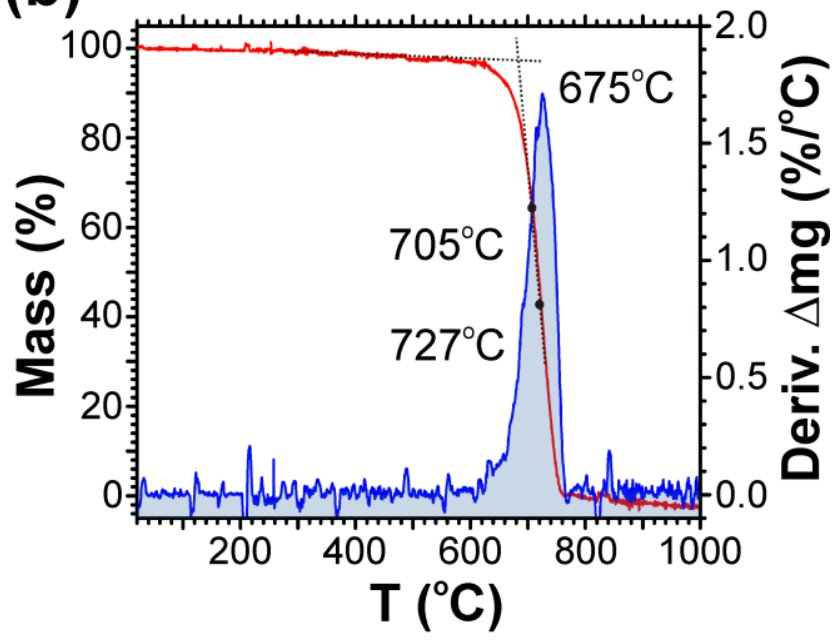

(c)

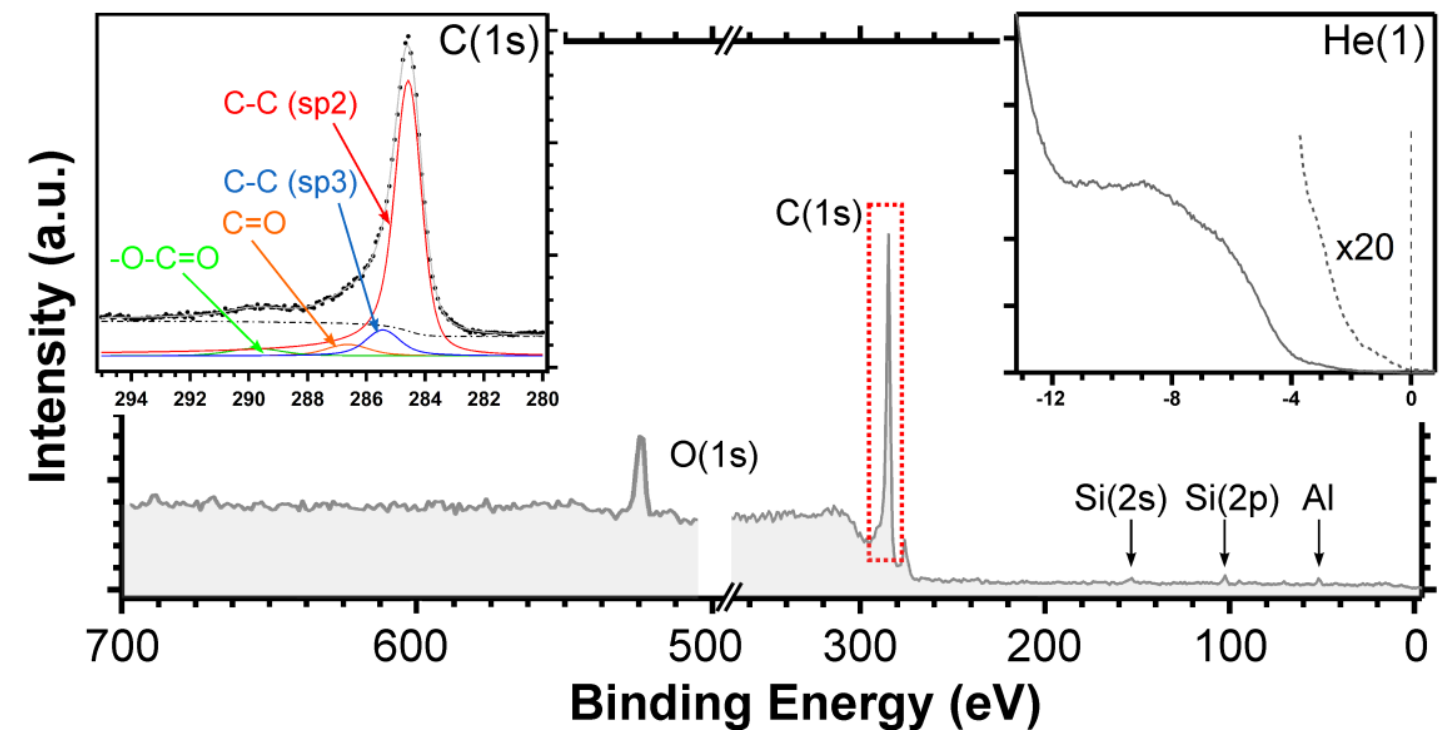

Figure S2. (Colour online). (a) TGA spectrum evidencing the high purity of the as-synthesised MWCNTs. (b) Typical EDX spectrum. Inset: Spatially resolved EDX plots showing the homogenous distribution of the $\mathrm{C}$ (88.4 at.\%), O (10.4 at.\%), Si (0.4 at.\%), and $\mathrm{Al}(0.2$ at.\%) (Scale bar: $300 \mathrm{~nm}$ ). Note the $\mathrm{Cu}$ peaks are associated with the supporting TEM grid. (c) XPS / UPS survey scan. Inset: Normalised detail of the $\mathrm{C}(1 \mathrm{~s})$ and the $\mathrm{He}(1)$ valence band peaks substantiating a plasma edge at $\sim 2.5 \mathrm{eV}$. Vertical dotted line denotes the Fermi edge. $\mathrm{C}(1 \mathrm{~s})$ Doniach-Sunjic and Guassian fits to the C-C $\left(s p^{2}\right)(284.5 \mathrm{eV}), \mathrm{C}-\mathrm{C}\left(s p^{3}\right)(285.4 \mathrm{eV}), \mathrm{CO}$ and $\mathrm{CO}_{2}$ components suggesting a $14-18 \% s p^{3}$ content, as suggested by direct UV Raman measurements. 